\title{
THE INTENSITIES OF SOME HYDROGEN, ARGON, AND HELIUM LINES IN RELATION TO CURRENT AND PRESSURE
}

\section{By P. G. Nutting and Orin Tugman}

Lines in the spectra of luminous gases have long been known to vary in intensity with conditions in the excited gas. Some gases exhibit two quite different spectra, the primary and secondary, either of which may predominate according to the violence of the discharge causing them. Sparks between metallic electrodes give spectra richer in lines than arcs between the same metals. Additional capacity in circuit intensifies certain lines, inductance weakens them. Similar variations might be cited in great number.

To study these complex variations in spectra we have chosen three typical spectra given by gases under known conditions-subject to control. Hydrogen was selected because its secondary spectrum persistently coexists with the primary even with feeble excitation and does not entirely displace it even with intense excitation. Argon gives a spectrum comparable with the spectra of many metals, intense excitation merely brings out a few additional lines. Helium is the opposite extreme from hydrogen; it persistently gives the same spectrum, no lines appearing or disappearing as the excitation is varied.

As spectral sources, we chose as the most reliable the Plücker tube operated on high potential (5000 and ro ooo volts) alternating current from transformers controlled by resistance in the low potential circuit. As the total fall of potential through our tubes was less than rooo volts, the current wave on 5000 or 10000 volt transformers was nearly sinusoidal. Some of the earlier work was repeated with direct current and gave the same values to I per cent. The first of the work was done with all-glass tubes with capillaries $2.6 \times 60 \mathrm{~mm}$ and disk electrodes in spherical bulbs. 
Such tubes carry a maximum current of from 20 to 50 milliamperes before showing sodium from the glass. Later we developed an end-on tube with a $3 \times 30 \mathrm{~mm}$ porcelain capillary and large cylindrical electrodes which would safely carry half an ampere of current. The bulbs of these tubes were made of a special Corning glass which will seal to porcelain.

The method of observation was to compare the intensities of the lines from the variable tube with those from another tube kept under constant conditions. All readings are therefore relative to some fixed current or pressure. Comparisons were made with a polarization spectrophotometer. The greatest care was observed in using only pure gases and in avoiding contamination, particularly by mercury.

To avoid possible errors due to varying temperature and deterioration of the capillary wall ( $V$. infra) the tubes were run on open circuit, lighting up only when a double key was pressed. Thus the tubes were spared unnecessary service and remained near room temperature. Precision milliammeters were continually in circuit with the tubes except when the disruptive discharge was used. Both ocular and collimator slits were opened to admit about $6 \mu \mu$ of the spectrum.

\section{HYDROGEN}

Observations were made at six different regions of the hydrogen spectrum, three in the primary and three in the secondary. The readings in the secondary were made on the prominent red, green, and blue lines, $\alpha 656, \beta 486$, and $\gamma 434 \mu \mu$, referred to collectively as $S$. In the primary spectrum no attempt was made to observe individual lines. A qualitative photographic test shows that the numerous fine lines vary sensibly together (except at very low pressures) and as they form, with low resolving power, a fairly uniform continuous spectrum, regions were selected for observation at about $6 \mathrm{II}, 545$, and $460 \mu \mu$, referred to as $a, b$, and $c$, and collectively as $P$.

The data taken on the variation of spectral intensity with current are given below. The first table refers to a tube with porcelain capillary $3 \mathrm{~mm}$ in diameter and $30 \mathrm{~mm}$ long viewed end on. 
TABLE I

\begin{tabular}{c|c|c|c|c|c}
\hline & & \multicolumn{3}{|c}{ H Line-Intensity $\div$ Intensity at $i=20, p=1 \mathrm{~mm}$} \\
\hline & No. obsns. & $S_{\alpha}$ & $S_{\beta}$ & $S_{\gamma}$ & $P_{a b c}$ \\
\hline & 2 & 1.0 & 1.0 & 1.0 & 1.0 \\
\hline 20 & 4 & 2.6 & 2.2 & 2.0 & 1.7 \\
40 & 1 & 3.9 & 2.6 & 2.9 & 2.3 \\
50 & 1 & 5.4 & 3.9 & 2.7 & 3.3 \\
75 & 3 & 14.2 & 10.2 & 5.5 & 4.4 \\
120 & 2 & 20.5 & 17.0 & 6.5 & 7.1 \\
200 & 5 & 29.2 & 18.1 & 10.7 & 8.2 \\
250 & 3 & 48.8 & 18.2 & 16.3 & 8.9 \\
300 & 1 & 57.0 & 26.5 & 14.0 & 11.5 \\
370 & 2 & 59.5 & 24.0 & 12.0 & 10.7 \\
380 & 2 & $\ldots . .5$ & $\ldots$. & $\ldots$. & 13.0 \\
400 & 2 & 66.0 & 26.5 & $\ldots .$. & 11.1 \\
440 & & & & & \\
\hline
\end{tabular}

All values for light intensity are relative to the intensity at $i=20$. A zero reading $(i=20)$ was taken just before and just after each reading at higher current and if the zero showed any shift the reading was rejected. Both tubes were filled with fresh gas at I mm pressure for each set of observations. The primary spectrum showed no selective (wave length) effect at even the highest currents, so the mean of the three readings is given in the last column.

These data are represented in Fig. I. The red line is seen to vary much more rapidly with current than the others. A least square reduction for the intensity $P$ of the primary spectrum gave

$$
\frac{P}{P_{20}}=1.042 \frac{i}{20}-0.0432\left(\frac{i}{20}\right)^{2}+0.00098\left(\frac{i}{20}\right)^{3}
$$

Calling the intensity of any line $L$ we computed values of $K$ in $\log \left(L / L_{0}\right)=K \log (i / 20)$. The values $K$ were very nearly constant for each of the lines over the wide range of current used, but showed a slight and systematic lowering for intermediate currents ( $50-200 \mathrm{~m} . \mathrm{a}$.). Since the total power absorbed by the gas is the product of potential gradient $(X)$ and current, we should expect 
line intensities to be simpler functions of power $X i$ than of current $i$. The gradient was determined and values are given below. Different lines should, however, be the same functions of gradient

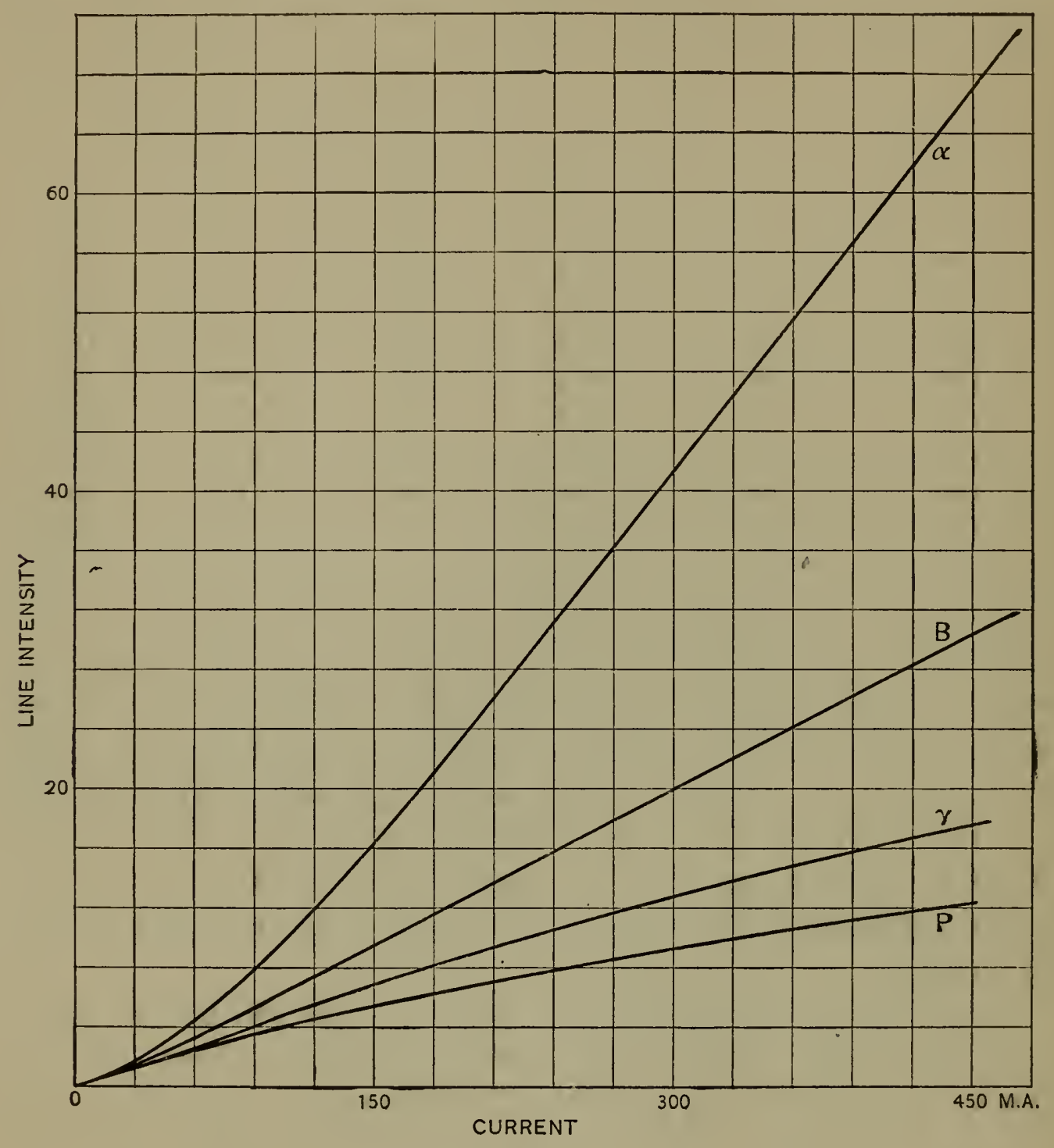

Fig. 1

and current. Taking the primary spectrum as a standard, we computed $m$ in

$$
\log \frac{\alpha}{\alpha_{20}}=m \log \frac{P}{P_{20}}
$$

and similarly for the lines $\beta$ and $\gamma$ and obtained values of $m$ showing no systematic variation with intensity. This is significant, considering the wide range of intensities covered. 
We find

Hence

$$
m_{a}=\mathrm{I} .67, m_{\beta}=\mathrm{I} .35, m_{r}=\mathrm{I} . \mathrm{I} 4 \pm .02
$$

$$
\left(\frac{\alpha}{\alpha_{20}}\right)^{.599}=\left(\frac{\beta}{\beta_{20}}\right)^{.729}=\left(\frac{\gamma}{\gamma_{20}}\right)^{.875}=\frac{P}{P_{20}}
$$

The computed uncertainty in $m$ is about 2 per cent. It may be expressed as a function of wave length $\lambda$ in

$$
\begin{aligned}
m & =68 \mathrm{I}\left(\frac{\mathrm{I}}{25 \mathrm{I}}-\frac{\mathrm{I}}{\lambda}\right) \\
m-\mathrm{I} & =68 \mathrm{I}\left(\frac{\mathrm{I}}{397}-\frac{\mathrm{I}}{\lambda}\right) \\
(m-\mathrm{I})_{a, \beta, r} & =\frac{2}{3}(\mathrm{I}, \mathrm{I} / 2, \mathrm{I} / 4---)
\end{aligned}
$$

In Fig. 2 are plotted these values of $m$ together with the relative energy intensities of the observed lines.

The curve for $m(\lambda)$ indicates that the fourth secondary line $(\delta, \lambda=4$ IO) would vary at the same rate $(m=1)$ as the primary spectrum, while lines farther out toward the head of the Balmer series would increase less rapidly than the primary with increase of current and hence could not be brought out prominently at this gas density with any current.

The most probable values of the spectral intensity taken from the adjusted curves are given in Table 2 .

TABLE II

\begin{tabular}{r|r|r|r|r}
\hline \multicolumn{1}{c|}{$i$} & $P$ & ${ }^{\prime}$ & $\beta$ & $\gamma$ \\
\hline 20 & 1.00 & 1.00 & 1.00 & 1.00 \\
50 & 2.1 & 3.45 & 2.72 & 2.33 \\
100 & 3.8 & 9.3 & 6.1 & 4.6 \\
150 & 5.4 & 16.7 & 9.7 & 6.8 \\
200 & 6.8 & 24.5 & 13.3 & 8.9 \\
250 & 8.2 & 33.5 & 17.1 & 11.0 \\
300 & 9.4 & 42.0 & 20.5 & 12.9 \\
350 & 10.4 & 49.8 & 23.5 & 14.4 \\
400 & 11.5 & 59.1 & 27.0 & 16.2 \\
450 & 12.3 & 66.1 & 29.5 & 17.4 \\
\hline
\end{tabular}


The relative energies of the given lines given in Table 3 and plotted in Fig. 2 were obtained by comparison with a carbon fila-

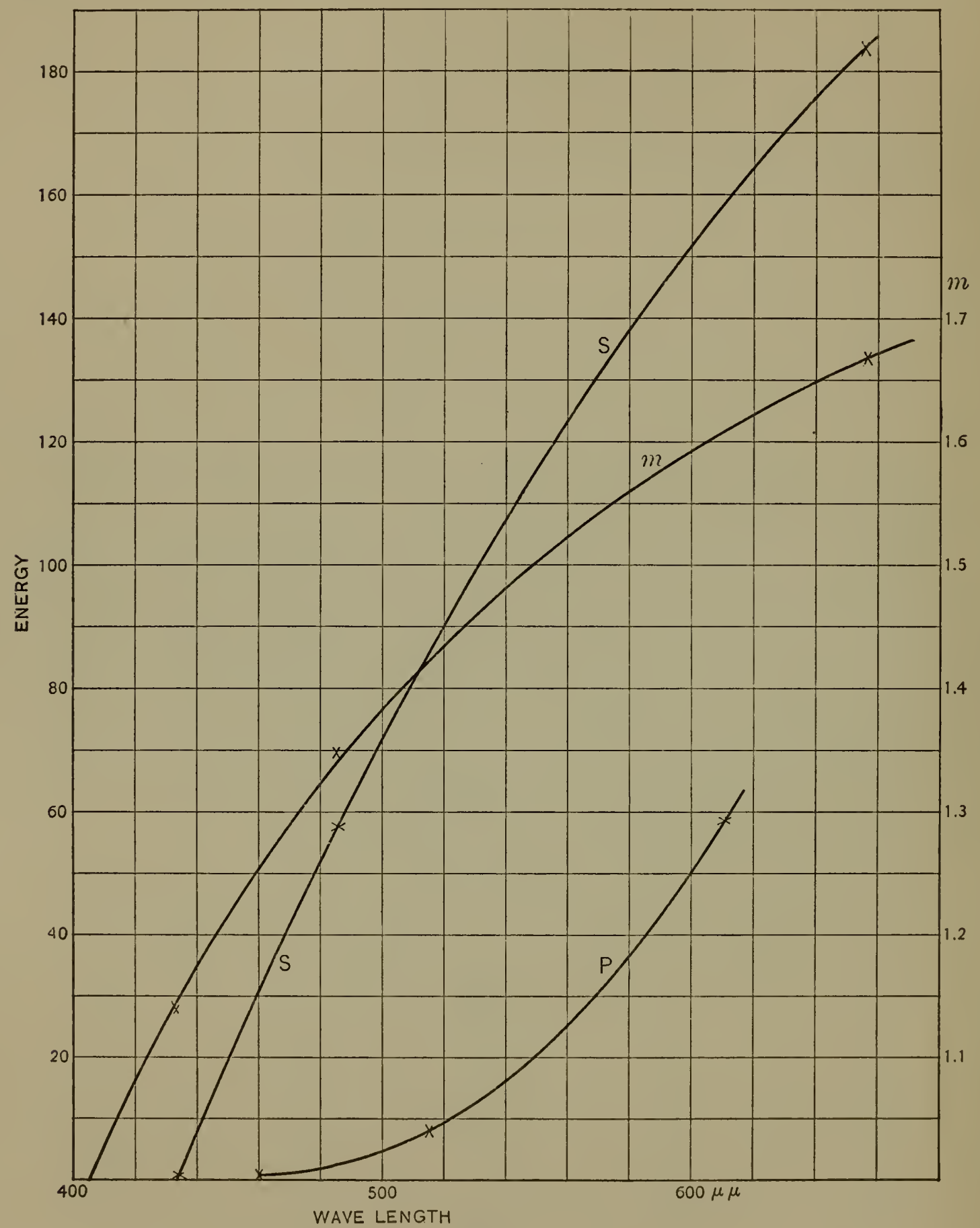

Fig. 2

ment glow-lamp whose energy-curve at a given voltage had been determined by Dr. Coblentz.

The uncertainty in the data given is perhaps 5 per cent. 
TABLE III

\begin{tabular}{c|c|c|c}
\hline Line & $\lambda$ & Lamp E & Hyd. E \\
\cline { 1 - 2 }$\beta$ & 656 & 197 & $184\left(\mathrm{E}_{\gamma}=1\right)$ \\
$\beta$ & 486 & 24 & $58\left(\mathrm{E}_{\gamma}=1\right)$ \\
$\gamma$ & 434 & 7.0 & 1 \\
$a$ & 611 & 130 & $59.7\left(\mathrm{E}_{c}=1\right)$ \\
$\delta$ & 515 & 38 & $8.3\left(\mathrm{E}_{c}=1\right)$ \\
$c$ & 460 & 14 & 1.0 \\
\hline
\end{tabular}

Considerable preliminary work was done with tubes having glass capillary portions of $2.6 \mathrm{~mm}$ bore viewed transversely. Data thus obtained are a useful supplement to that (above) obtained with porcelain capillary and heavy currents. In Table 4 are combined data taken with six different new tubes all of the same dimensions and each filled with fresh gas at I $\mathrm{mm}$ pressure. As neither primary nor secondary spectrum showed any selective effect, the results for each spectrum are combined.

TABLE IV

\begin{tabular}{|c|c|c|c|c|c|c|c|}
\hline Current & 2 & 3 & 5 & 7 & 9 & 12 & 15 \\
\hline Primary & 0.16 & 0.24 & 0.34 & 0.49 & 0.65 & 0.81 & 1.0 \\
\hline Secondary & .04 & .09 & .17 & .29 & .42 & .65 & 1.0 \\
\hline
\end{tabular}

Writing as before $S / S_{15}=\left(P / P_{15}\right)^{m}, m=1.73 \pm .02$, independent of current as with large currents.

This lack of selective effect was at first attributed to lack of saturation in the thin column viewed from the side, but careful determinations at Io m. a. made with the end-on porcelain capillary showed that there was no selective effect as large as one-third that indicated by extrapolation from higher currents.

The smallest currents at which observations were taken were 2 m.a. Fig. 3 shows that all lines vanish at or very near zero current, not at a finite value of the current as has been frequently assumed. The cause of the whiteness of the glow at low-current densities and redness at high is readily seen from Figs. I and 3 .

The potential gradient (volts per $\mathrm{cm}$ ) in the tubes with glass capillary $2.6 \mathrm{~mm}$ diameter is given in the following table. It was 
determined by measuring the total drop between electrodes of a tube with a long capillary before and after cutting out about Io $\mathrm{cm}$ of the capillary.

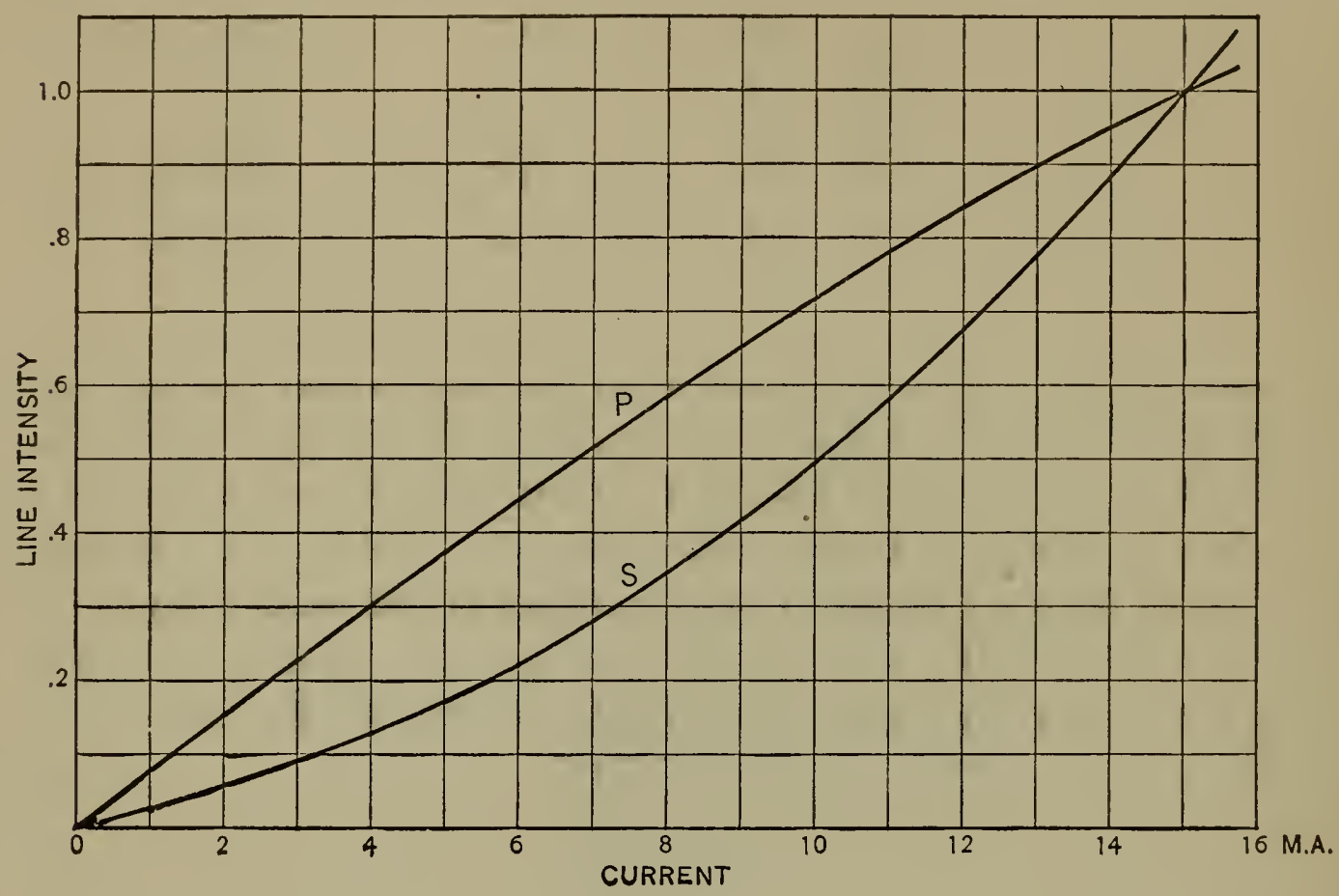

Fig. 3

Volts per $\mathrm{cm}$ in $\mathrm{H}, 2.6 \mathrm{~mm}$ Capillary

\begin{tabular}{c|c|c|c|c|c}
\hline & \multicolumn{5}{|c}{ Pressure (mm) } \\
\cline { 2 - 6 } & 0.5 & 1.0 & 2.0 & 3.0 & 8.0 \\
\hline 2 & 44.5 & 60.2 & 97.0 & 125 & 191 \\
5 & 37.8 & 56.1 & 90.4 & 107 & 150 \\
9 & 33.7 & 52.1 & 81.1 & 93 & 128 \\
12 & 34.2 & 50.5 & 74.6 & 84 & 122 \\
15 & 35.8 & 49.5 & 67.4 & 78 & 120 \\
\hline
\end{tabular}

Finally, the gradient in the porcelain capillary of the large tube was determined by measuring the drop between potential electrodes inserted at each end of the porcelain part.

Volts per $\mathrm{cm}$ in $\mathrm{H}, 3 \mathrm{~mm}$ cap., $1 \mathrm{~mm}$ Pressure

\begin{tabular}{l|r|r|r|r|r|r|r|r|r|r}
\hline Current & 20 & 50 & 100 & 150 & 200 & 250 & 300 & 350 & 400 & 450 \\
Volts/cm & 50.0 & 46.0 & 43.3 & 41.7 & 40.7 & 40.0 & 39.7 & 39.3 & 39.0 & 38.7 \\
\hline
\end{tabular}


Expressing line intensity, $L$, as a function of internal energy $X i=E$

$$
\frac{L}{L_{0}}=\left(\begin{array}{c}
E \\
E_{0}
\end{array}\right)^{n}
$$

$\begin{array}{lcccc}\text { Line } & P & \alpha & \beta & \gamma \\ n= & .90 \mathrm{I} & \mathrm{I} .500 & \mathrm{I} .216 & \mathrm{I} .022 \pm .005\end{array}$

without systematic variation. The relation between $m$ and $n$ is $n=0.90 \mathrm{I} m$.

The above empirical law is consistent with a radiation function of the form

$$
L=C_{1} \Lambda E^{\kappa}=C_{1} \Lambda e^{\kappa T}
$$

in which $\Lambda$ and $\kappa$ are functions of wave length, and $T$ is a function of internal energy.

\section{GAS-DENSITY AND OTHER EFFECTS}

The effect of pressure on line-intensity at constant current was studied with glass tubes similar to those last described. From several hundred observations the following mean values were adopted. No selective effect was apparent in either primary or secondary, but these differ considerably from each other. All values are in terms of those at I mm pressure as unity.

\begin{tabular}{l|r|r}
\hline Pressure & Secondary & Primary \\
\hline $0.25 \mathrm{~mm}$ & 0.55 & $\ldots$. \\
0.50 & .78 & 0.80 \\
0.75 & .97 & .97 \\
1.00 & 1.00 & 1.00 \\
1.5 & .90 & .92 \\
2.0 & .78 & .85 \\
3 & .60 & .72 \\
4 & .47 & .61 \\
5 & .38 & .53 \\
6 & .32 & .48 \\
7 & .28 & .44 \\
8 & .25 & .41 \\
10 & .20 & .38 \\
\hline
\end{tabular}


The current was $15 \mathrm{~m} . \mathrm{a}$. (tubes in series) and the reference tube filled at I $\mathrm{mm}$ pressure in each case. These results are shown graphically in Fig. 4.

With both tubes filled with hydrogen at $0.3,0.5,2$, and $8 \mathrm{~mm}$ pressure and a current of $15 \mathrm{~m}$.a. through the reference tube, readings were taken with variable current ( 2 to 15 m.a.) through the observation tube. These sets of readings differed very little from those taken at I $\mathrm{mm}$ pressure.

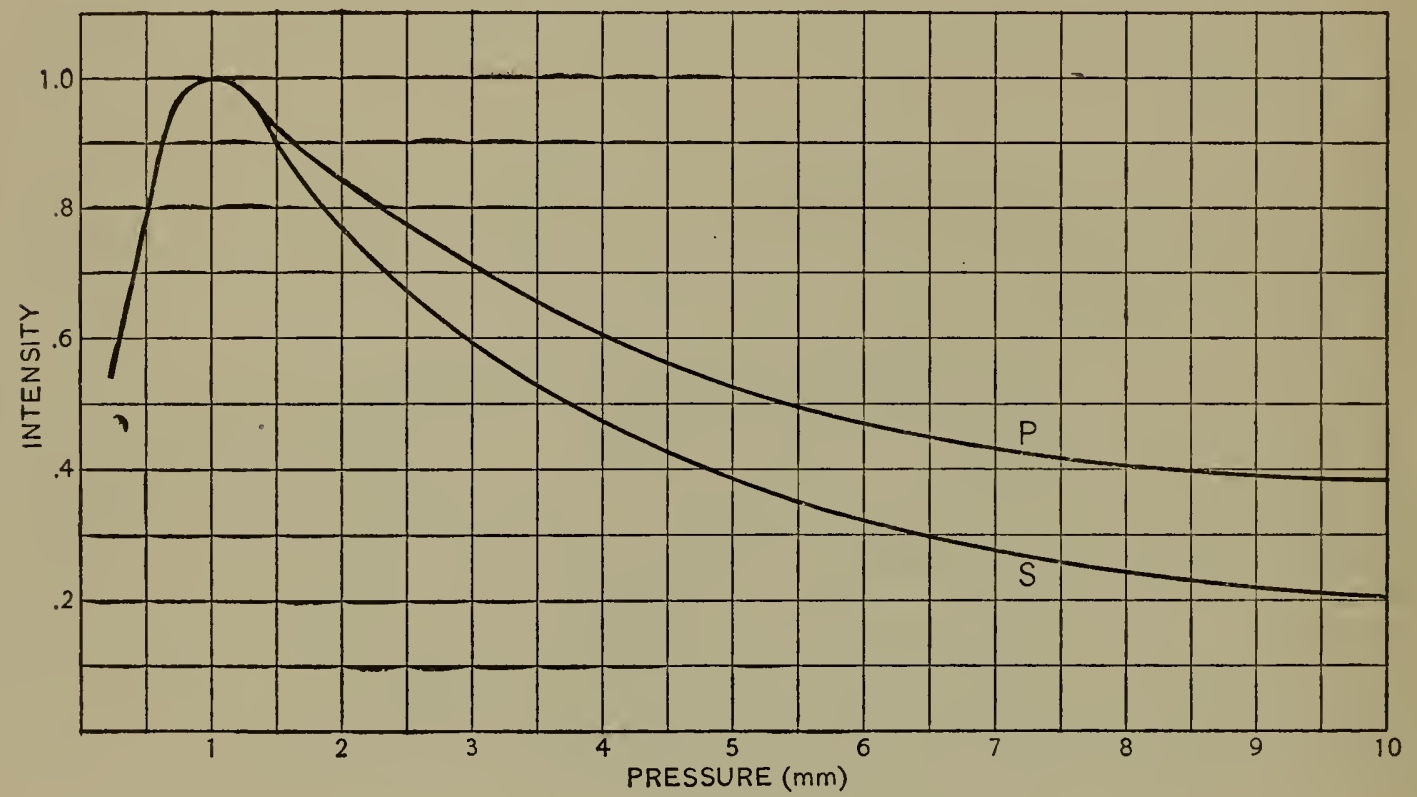

Fig. 4

Temperature of wall.-When the capillary part of a tube was heated with a flame to about $200^{\circ}, \alpha$ fell off 20 to 30 per cent, while $\beta$ and $\gamma$ and the whole of the primary were not affected by as much as 3 per cent. The tube recovers as it cools and the effect was repeated on several tubes. Since the current itself warms the tube $20^{\circ}$ to $100^{\circ}$, very discordant results may be obtained unless the tubes are maintained at constant temperatures.

Effect of use.-A tube of hydrogen after half an hour of continuous operation shows a marked decrease in intensity of all lines of both spectra. After an hour's run, a purple tinge may be detected in the capillary, and if this be cut out of the tube and viewed end-on, a layer of glass less than $0.2 \mathrm{~mm}$ thick, next to the inner wall, will be seen to be highly colored. This coloring is not 
affected by moderate heating, but is completely removed by heating to the softening point of the glass; the effect increases steadily with continued use. Refilling a tube with fresh gas at the same pressure produces no measureable effect on spectral intensity.

This effect on the inner wall might be due either to negative electron bombardment or to ionization by the extreme ultra-violet waves in which the hydrogen spectrum is so rich. We are inclined to the latter view. The effect is greater for hydrogen than any other gas. A helium tube has been run a hundred hours without appreciable deterioration at a current that would have produced a ro per cent change in a hydrogen tube in half an hour.

Oxygen with hydrogen.-Both reference tube and working tube were filled with hydrogen at $2 \mathrm{~mm}$ pressure, then zero readings were taken, the reference tube closed off, and an additional $2 \mathrm{~mm}$ of oxygen admitted to the working tube. Then with tubes in series (I 5 m.a. through both) it was found that the oxygen had cut down $\alpha, \beta$, and $\gamma$ about 30 per cent each, and $a, b$, and $c$ considerably more than that amount, and $a$ more than $c$ so that the red primary barely showed. The net effect of the oxygen then is to make the secondary spectrum much more prominent. It is easy to see why this spectrum has so often been attributed to water-vapor. This is a rich field for further study; we have touched upon it only to guard against possible error.

In the cathode glow and striations the relation of primary to secondary varies from that in the ordinary anode column. The primary is relatively brighter ( 50 per cent) in the brighter parts of the striations where recombination is supposed to be particularly active. In an ordinary tube with disk electrodes and plenty of room for the cathode glow, the secondary is slightly more prominent in that glow than in the capillary; but in other tubes with cylindrical electrodes set close to the walls, the cathode's glow is nearly white and strongly favors the primary.

At atmospheric pressure a zinc spark $(2 \mathrm{~mm})$ in hydrogen gave hydrogen spectra not very different nor in different proportion from those in the comparison tube. The increased width of the lines, however, made a more exact comparison difficult.

A tube with fine capillary (about $0.3 \mathrm{~mm}$ ) when adjusted to the same current-density as the reference tube gave spectra so nearly 
like those of the reference tube that no certain differences could be detected.

End-on effect.-Using the light from the side of a $2.6 \mathrm{~mm}$ capillary, we compared it with an end-on tube with a capillary portion $3.2 \times 32 \mathrm{~mm}$. This gave for relative intensities:

$\begin{array}{cccccrr} & \alpha & \beta & \gamma & a & b & c \\ \text { End-on } & \text { I } & \text { I.35 } & \text { I.58 } & 4.37 & 4.37 & 4.39\end{array}$

These are relative to $\alpha$, since there was an indeterminate constant factor involved. This apparently great departure from saturation may be due to a difference in the electrical conditions as the current leaves a capillary. We later obtained similar readings (not quite as high) from one end of a capillary viewed from the side.

Spark, capacity, and inductance.-The disruptive discharge was studied in considerable detail with various tubes containing gas at I, 5, and ro $\mathrm{mm}$ pressure. Two identical tubes were adjusted to I 5 m.a. current, but on independent circuits, and then the discharge in one of them made disruptive, the reference tube remaining at I $\mathrm{mm}$ and $\mathrm{I} 5 \mathrm{~m} . \mathrm{a}$. in each case. Some typical results are given below, the three primary readings being combined, as they remained equal throughout to within the uncertainty of measurement.

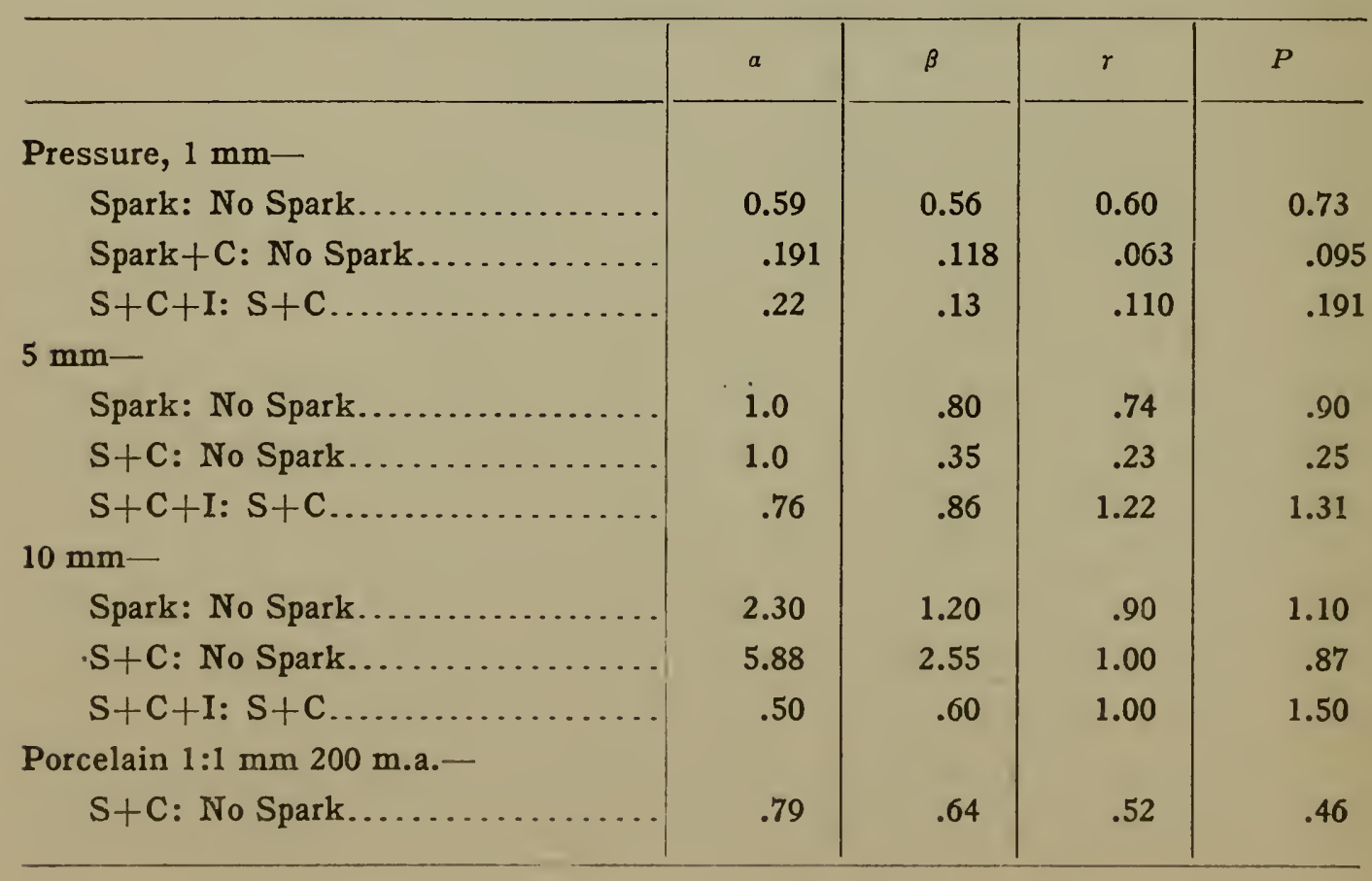


These results show the same selective effects as were obtained with heavy steady currents. In the first case, for example, the effect of capacity was to give $\alpha, \beta$, and $\gamma$ the ratio $3: 2:$ I while $P$ lies between $\beta$ and $\gamma$. These agree well with the values for a current of 200 to $250 \mathrm{~m}$.a. in the porcelain tube. On the other hand, in the last case where a heavy current (200 m.a.) was used with the condenser, the effect was much less marked, about equivalent to raising the current to $300 \mathrm{~m}$.a. The apparent weakening of the discharge was due to the longer interval in which no current is passing. The effect of inductance appears to be merely to neutralize part of the capacity effect. The secondary lines $\alpha, \beta$, and $\gamma$ are affected by both capacity and inductance in the order named, $\alpha$ most and $\beta$ least.

Spectroheliographs taken with the red hydrogen line show distinctly higher levels of the solar atmosphere than those taken with the lines of shorter wave length. Our results on the effect of varying the intensity of excitation indicate just the opposite effect, the lower layers being more intensely excited should show the red line relatively more prominent than the outer layers. The selective effect of varying gas density we found to be very small over a range of pressures from $0.2 \mathrm{~mm}$ to I atmosphere, so that the observed solar effect could not plausibly be attributed to this cause. Kayser ${ }^{1}$ holds to the view that lines of the same spectral series should vary with excitation as would portions of the same wave lengths in the spectrum of a heated cavity, a view contradicted by our results with the hydrogen secondary spectrum.

The solar effect and the end-on effect above recorded may be accounted for very simply, however, by the blanketing effect of the outer layers of a radiating gas upon the inner layers. The chief result of this blanketing being that the fainter lines in the spectrum of the inner layers would be relatively brighter than in that from the outer layers.

\section{ARGON}

The change in the argon spectrum from the "red" to the "blue" as the discharge is made disruptive is well known. The change is due to the production of additional lines in the blue and violet. 
Thus the argon spectrum behaves like the spectra of most metals and is intermediate in behavior between spectra which do not vary at all and those which vary easily and completely. These additional lines of the secondary (blue) spectrum we were able to produce in the porcelain tube without the aid of series spark or capacity and their behavior was studied at currents ranging from $450 \mathrm{~m}$. a. down to about $100 \mathrm{~m}$. a.

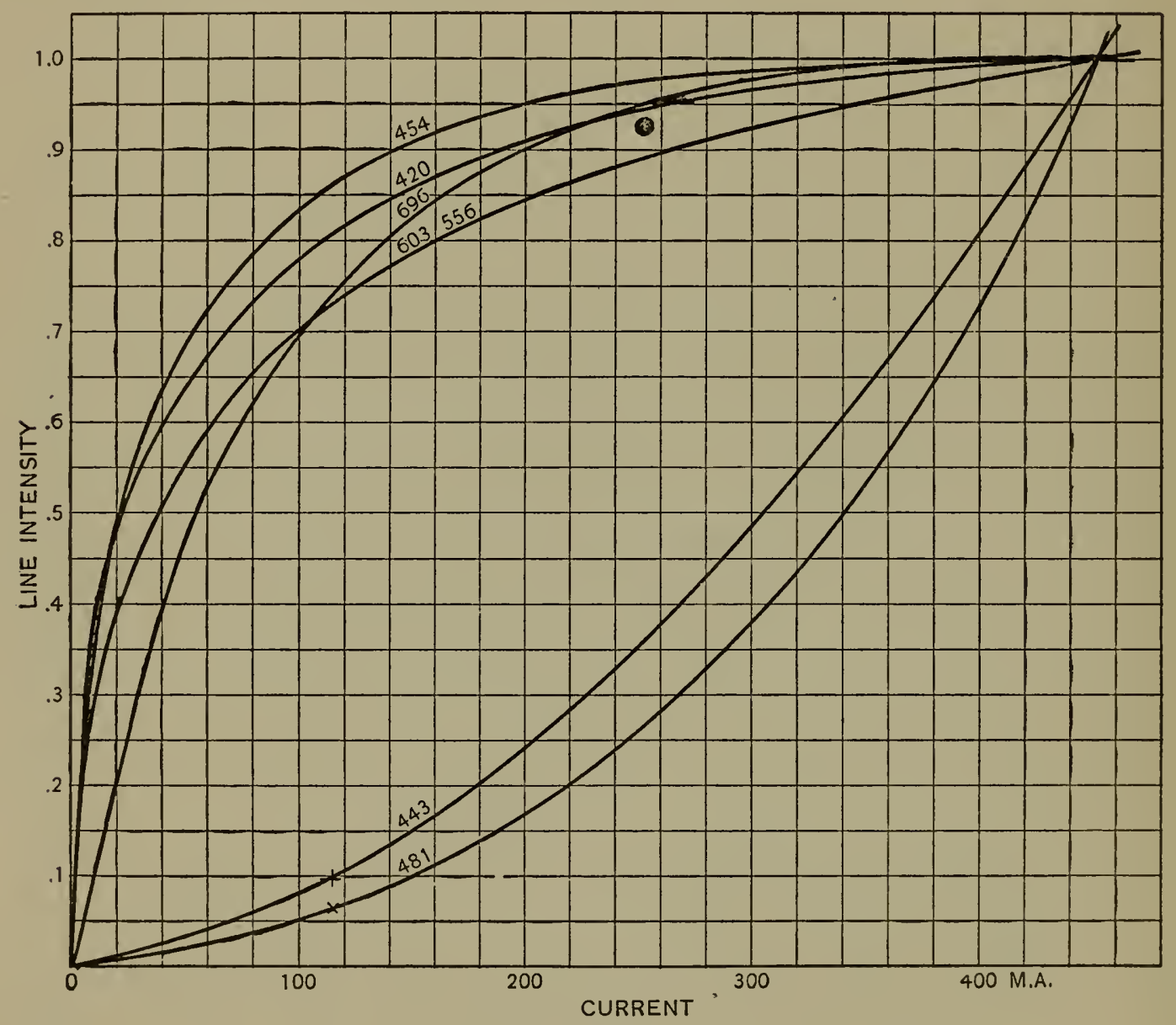

Fig. 5

We selected for study five of the most prominent lines of the primary (red) spectrum. The primary lines were: Red $\lambda 696$, orange $\lambda 60_{3}$, green $\lambda_{55} 6$, blue $\lambda_{454}$, and violet $\lambda_{420} \mu \mu$. The secondary groups studied were at $\lambda_{443}$ and $\lambda_{4} 8 \mathrm{r} u \mu$. These are in regions quite free from primary lines.

The data for argon are given in the following table and figure. The values given are graphical means of from two to six determinations at eight values of the current for the primary lines and 
five (I 5 to $450 \mathrm{~m}$. a.) for the secondary, using porcelain capillary $3 \mathrm{~mm}$ in diameter.

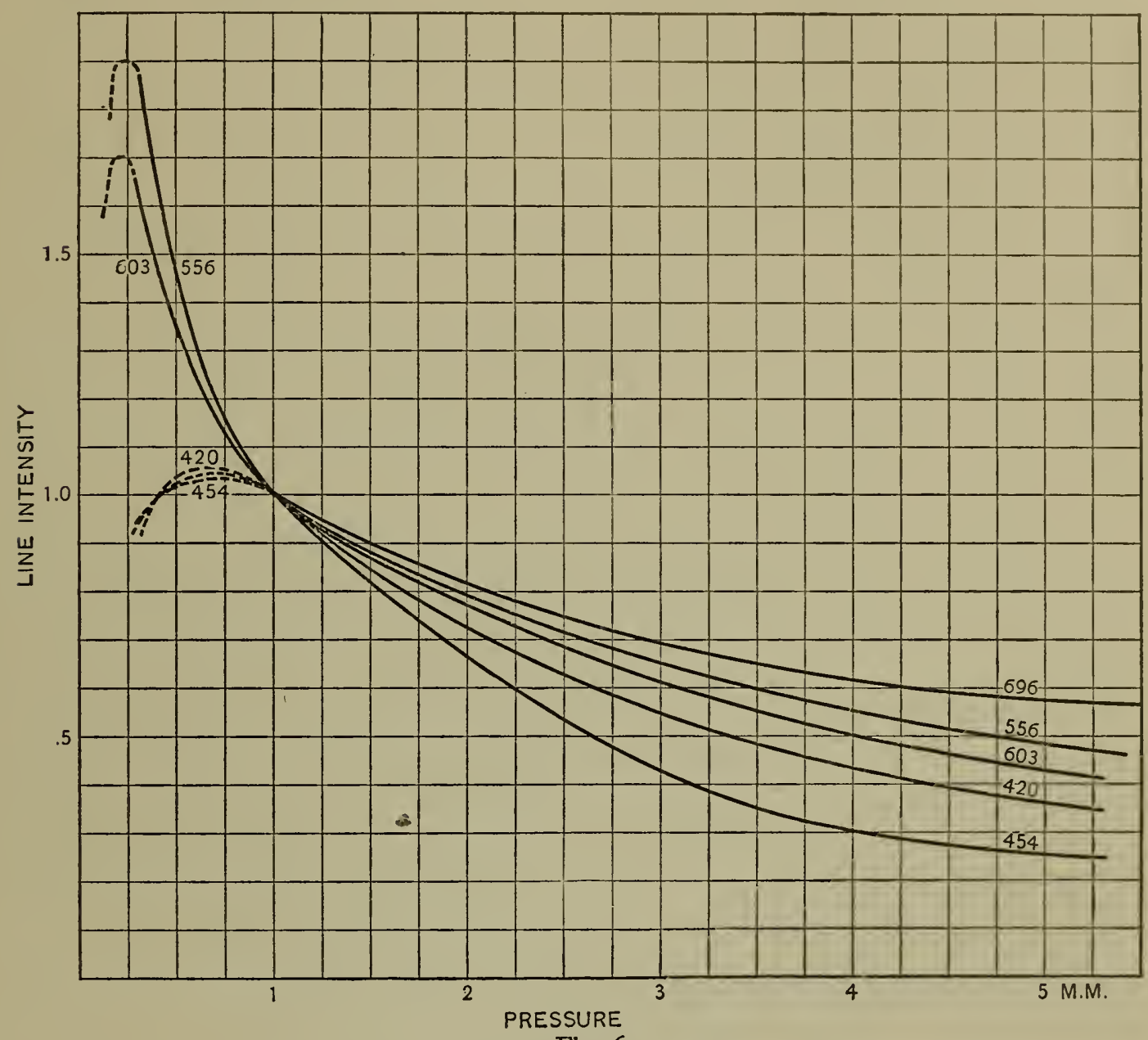

Fig. 6

Data on Argon

\begin{tabular}{|c|c|c|c|c|c|c|c|}
\hline \multirow{2}{*}{ Current } & \multicolumn{5}{|c|}{ Primary spectrum } & \multicolumn{2}{|c|}{ Secondary } \\
\hline & 696 & 603 & 556 & 454 & 420 & 481 & 443 \\
\hline 20 & 0.20 & 0.39 & 0.40 & 0.49 & 0.47 & $\ldots$ & $\ldots$ \\
\hline 50 & .47 & .54 & .55 & .68 & .62 & .... & $\ldots$ \\
\hline 100 & .69 & .70 & .68 & .83 & .77 & 0.050 & 0.082 \\
\hline 200 & .90 & .85 & .83 & .95 & .91 & .16 & .24 \\
\hline 300 & .98 & .92 & .92 & .98 & .97 & .37 & .49 \\
\hline 375 & $\ldots$ & $\ldots$ & $\ldots$ & .... & $\ldots$ & .62 & .74 \\
\hline 450 & 1.00 & 1.00 & 1.00 & 1.00 & 1.00 & 1.00 & 1.00 \\
\hline
\end{tabular}

The wide departures of line intensities from proportionality with current are well shown in Fig. 5. All the primary lines rise very 
abruptly and then become nearly constant at high currents, while the secondary groups rise very slowly at first and then very steeply. There is no indication that these secondary lines appear abruptly at a large value of the current, but rather that they start at or near zero of current. Empirical exponential relations between the intensities of the argon lines may be set up as for the hydrogen lines, but the uncertainty in the data is so great that such relations are of little value. No determinations of the potential gradient in argon were made.

With 20 m.a. current, when condenser with series spark was added, relative intensities of primary and secondary were such as were obtained at $250 \mathrm{~m}$.a. without capacity. With $200 \mathrm{~m}$.a. initial current, the effect of condenser was roughly equivalent to increasing the current to $350 \mathrm{~m}$.a.

In studying the effect of gas density on line intensity no attempt was made to adjust the pressure to any given value on account of the risk of introducing impurities. Sets of readings were taken first at high pressure, then after removal of a portion of the gas, and so on down to a cathode ray vacuum. This process was repeated six times and mean curves drawn through points located at the many different pressures. At pressures below I $\mathrm{mm}$ determinations differed so widely that the data in this region can be regarded as having but little more than qualitative value. Observations were made on glass tubes with $2.6 \mathrm{~mm}$ capillary viewed side on at the middle. The current was $20 \mathrm{~m} . \mathrm{a}$. throughout.

Argon, Line Intensity and Pressure

\begin{tabular}{c|c|c|c|c|c}
\hline & \multicolumn{5}{|c}{ Line } \\
\cline { 2 - 6 } & 696 & 603 & 556 & 454 & 420 \\
\hline 0.35 & 0.96 & 1.58 & 1.85 & 0.97 & 0.95 \\
.70 & 1.04 & 1.16 & 1.21 & 1.03 & 1.06 \\
1.00 & 1.00 & 1.00 & 1.00 & 1.00 & 1.00 \\
2.00 & .82 & .79 & .79 & .67 & .72 \\
3.00 & .70 & .66 & .66 & .43 & .55 \\
4.00 & .62 & .55 & .55 & .31 & .43 \\
5.00 & .58 & .49 & .49 & .37 & .25 \\
\hline
\end{tabular}

The blue and violet lines are most sensitive to pressure changes, the red line least. 


\section{HELIUM}

The helium spectrum is of the type that does not vary as the discharge is made disruptive. The cathode glow is richer in lines than the anode or capillary glow, and with disruptive discharge at low pressures these cathode lines appear in all parts of the discharge. Excluding these, we have not been able to obtain new

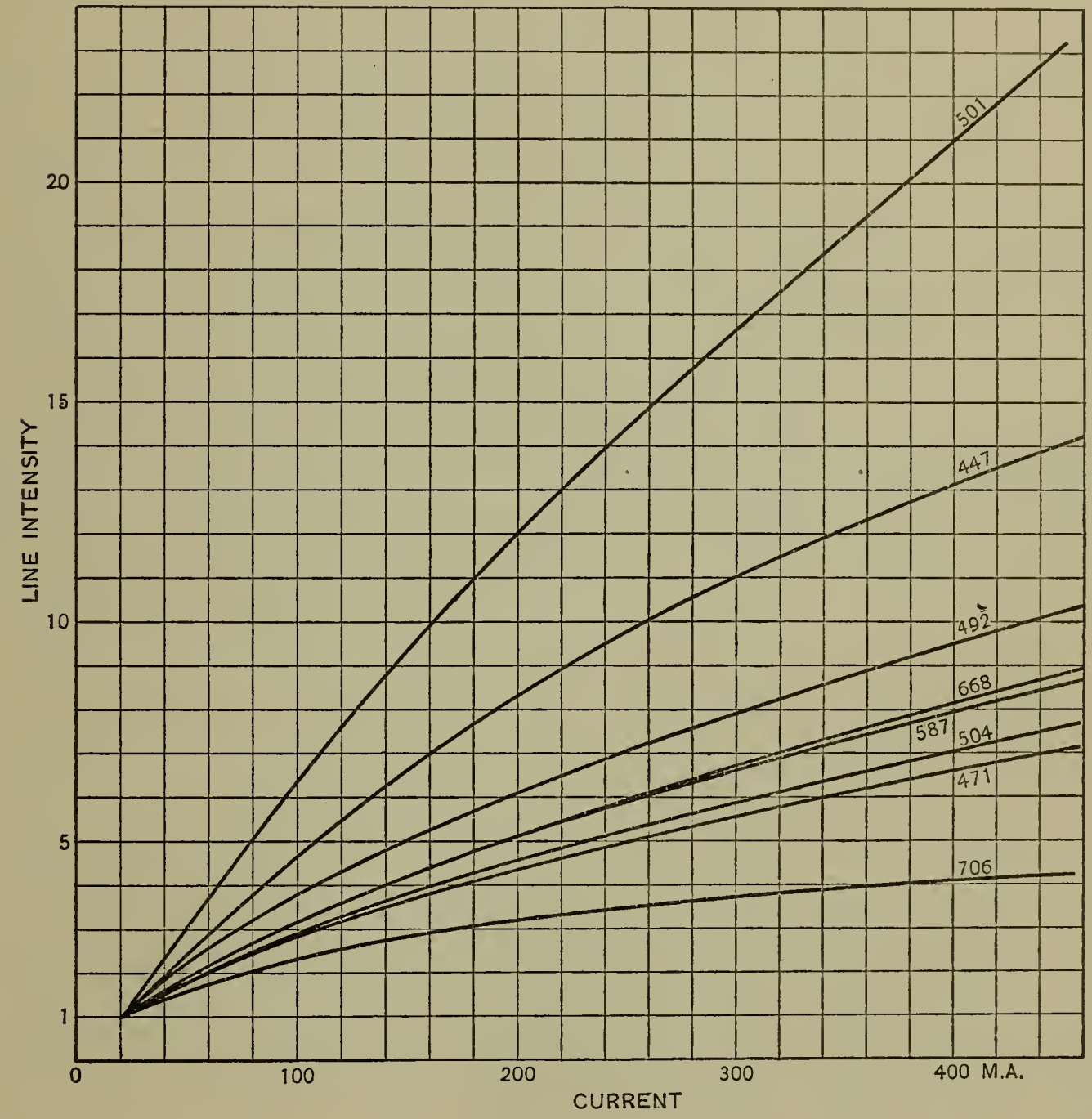

Fig. 7

lines with the heaviest currents and disruptive discharges available nor with pressures up to $10 \mathrm{~mm}$

The total light emission of helium per cm length of capillary as a function of current, pressure, bore, and form of current has been previously studied. ${ }^{1}$ Preliminary results ${ }^{2}$ on the red and blue

${ }^{1}$ Bull. Bu. of Standards, 4, pp. 5II-523; I908. $\quad{ }^{2}$ Natıre, 81, p. I89; I909. 
lines 668 and 447 showed that these varied with current in sensibly the same proportion when this variation was eighteenfold, the current being varied from 2.5 to $45 \mathrm{~m}$. a. in a $2.6 \mathrm{~mm}$ capillary. We have since extended this work to eight lines and to currents of $450 \mathrm{~m}$. a. in the $3 \mathrm{~mm}$ porcelain capillary.

The lines studied were the eight brightest in the visible spectrum; the two red 706 and 667 , the yellow 587 , the three green 504,501 , and 492 , blue $47 \mathrm{I}$ and $447 \mu \mu$. The variation of line intensity with current is given in the following table. These data are graphical means of six sets of observations at seven values of the current. They were obtained with new tubes with porcelain capillary $3 \mathrm{~mm}$ in diameter and $30 \mathrm{~mm}$ long viewed end on. Another similar tube, which had previously been used for a month with argon, gave curves ro per cent lower but of the same form.

Helium. Intensity-Current Data

\begin{tabular}{c|c|c|c|c|c|c|c|c}
\hline Current & 706 & 667 & 587 & 504 & 501 & 492 & 471 & 447 \\
\cline { 2 - 4 } 20 & 1.0 & 1.0 & 1.0 & 1.0 & 1.0 & 1.0 & 1.0 & 1.0 \\
\cline { 2 - 5 } 50 & 1.5 & 1.9 & 1.9 & 1.8 & 3.0 & 2.2 & 1.7 & 2.4 \\
100 & 2.3 & 3.1 & 3.1 & 2.9 & 6.3 & 3.8 & 2.8 & 4.6 \\
200 & 3.1 & 5.1 & 5.1 & 4.5 & 12.1 & 6.1 & 4.7 & 8.3 \\
300 & 3.7 & 6.7 & 6.6 & 5.9 & 16.6 & 7.9 & 5.5 & 11.0 \\
450 & 4.2 & 8.8 & 8.5 & 7.6 & 23.1 & 10.2 & 7.1 & 14.1 \\
\hline
\end{tabular}

All lines increase less and less rapidly with increase in current (and energy), thus showing the characteristic of primary lines.

The potential gradient (volts/cm) in helium at various pressures and currents was determined with one of the $3-\mathrm{mm}$ porcelain capillary tubes used in determining the intensity-current curves. The results are given below. 
Volts per $\mathrm{cm}$ in He. $3 \mathrm{~mm}$ Capillary

\begin{tabular}{c|c|c|c|c|c|c|c|c}
\hline \multirow{2}{*}{$\begin{array}{c}\text { Pressure } \\
(\mathrm{mm})\end{array}$} & \multicolumn{8}{|c}{ Current (m. a.) } \\
\cline { 2 - 7 } & 5 & 10 & 20 & 50 & 100 & 200 & 300 & 450 \\
\hline & 29.8 & 28.8 & 27.4 & 25.3 & 23.4 & 23.3 & 21.6 & 21.0 \\
2 & 30.5 & 29.8 & 28.9 & 26.8 & 24.8 & 23.4 & 22.9 & 22.5 \\
4 & 31.3 & 30.7 & 29.8 & 28.0 & 26.5 & 24.8 & 24.2 & 23.7 \\
5 & 32.2 & 31.7 & 30.5 & 28.9 & 27.1 & 25.4 & 24.7 & 24.3 \\
6.6 & 33.7 & 32.7 & 31.7 & 29.8 & 28.2 & 26.2 & 25.3 & 24.7 \\
10.0 & 35.7 & 35.0 & 34.3 & 32.7 & 30.7 & 28.1 & 27.5 & 27.0 \\
\hline
\end{tabular}

The complete data on change of intensity with pressure are given in the following table. About forty observations were made on each line, using different tubes and different fillings of gas. All the data given refer to glass tubes with $2.6-\mathrm{mm}$ capillary carrying $20 \mathrm{~m} . \mathrm{a}$. current (variable and reference tubes in series) viewed from the side at the middle. We also investigated the effect of pressure, using capillaries of $\mathrm{I}-\mathrm{mm}$ and of $5-\mathrm{mm}$ bore, and found the variation with pressure much less in the former case and slightly enhanced in the latter (in comparison with 2.6-mm tubes).

Helium. Pressure Effect

\begin{tabular}{|c|c|c|c|c|c|c|c|c|}
\hline \multirow{2}{*}{$\begin{array}{l}\text { Pressure } \\
\text { (mm } \mathrm{Hg})\end{array}$} & \multicolumn{8}{|c|}{ Wave lengths of lines } \\
\hline & 706 & 667 & 587 & 504 & 501 & 492 & 471 & 447 \\
\hline 1 & ..... & 0.8 & ... & 2.6 & 1.08 & 1.0 & 1.2 & 0.7 \\
\hline 1.5 & 0.7 & .92 & 0.5 & 2.3 & 1.20 & 1.12 & 1.4 & .9 \\
\hline 2 & .82 & 1.02 & .8 & 2.0 & 1.28 & 1.20 & 1.4 & 1.05 \\
\hline 3 & 1.05 & 1.1 & 1.1 & 1.6 & 1.28 & 1.22 & 1.35 & 1.18 \\
\hline 4 & 1.02 & 1.1 & 1.1 & 1.25 & 1.15 & 1.12 & 1.15 & 1.15 \\
\hline 5 & 1.00 & 1.00 & 1.00 & 1.00 & 1.00 & 1.00 & 1.00 & 1.00 \\
\hline 6 & .97 & .95 & .95 & .85 & .90 & .87 & .88 & .90 \\
\hline 7 & .93 & .90 & .90 & .72 & .80 & .75 & .75 & .79 \\
\hline 8 & .88 & .85 & .87 & .62 & .70 & .68 & .70 & .70 \\
\hline 9 & .82 & .80 & .85 & .55 & .65 & .60 & .60 & .65 \\
\hline 10 & .8 & .77 & .82 & .50 & .6 & .52 & .58 & .62 \\
\hline
\end{tabular}

The pressures at which the maximum emission occurs are higher for helium than for either hydrogen or argon. 


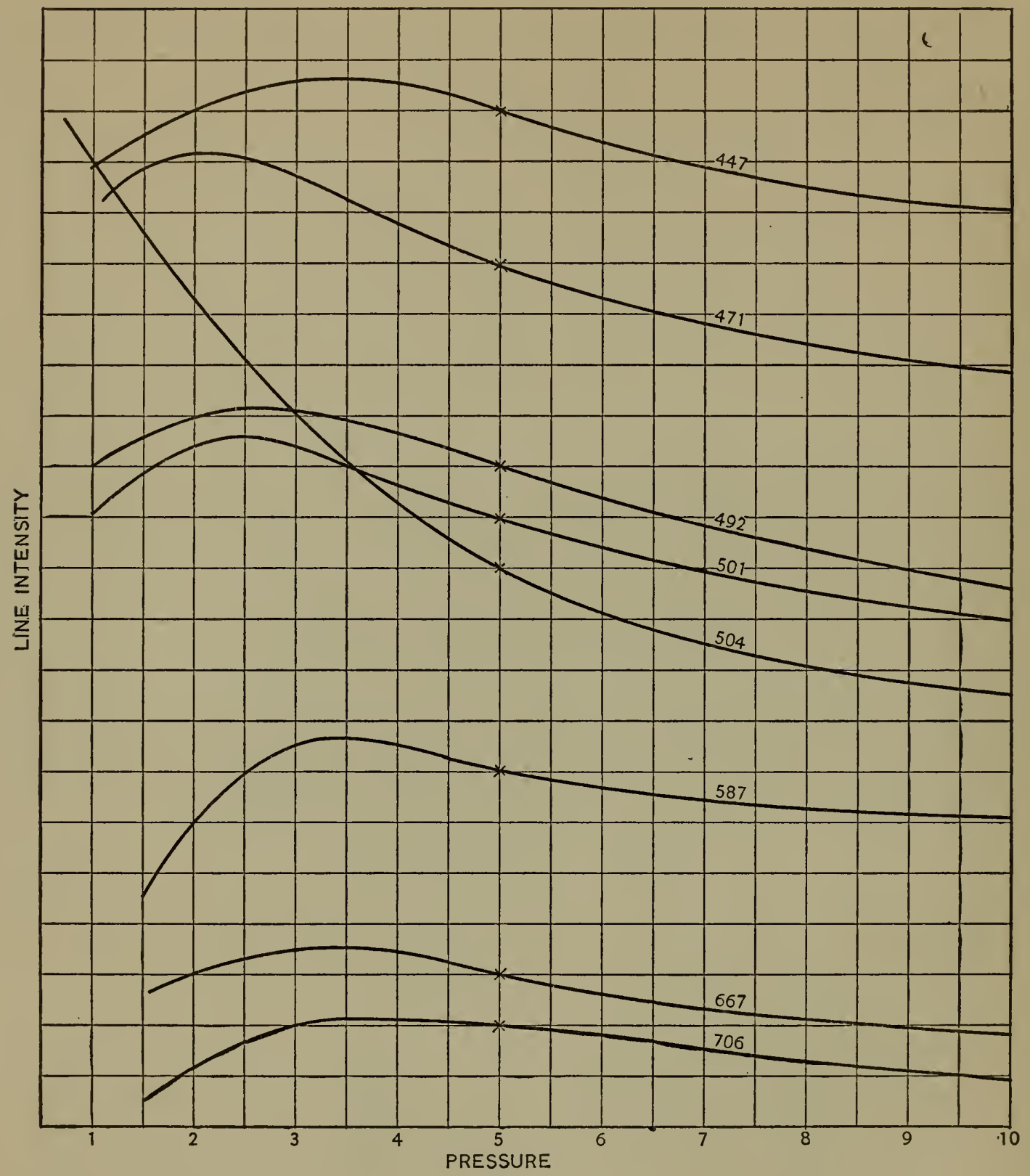

Fig. 8

DISCUSSION AND SUMMARY

This work has been done with photometer and alternating current rather than with bolometer and direct current, not only on account of the greater ease and simplicity of manipulation but on account of the much greater range of intensity that may be studied. The lower half of our curves of variation with current could hardly be obtained with bolometer or Rubens thermopile, and it is just this region that is of greatest importance. Again, in 
working with great current densities narrow capillaries are to be avoided on account of increased wall losses. We have used large capillaries $(2.6$ and $3.0 \mathrm{~mm}$ ) throughout, and yet we regard wall losses as the chief source of uncertainty in our results.

An essential source of ambiguity in all work of this nature lies in the uncertainty in the degree of saturation (radiation compared with the radiation from a very thick layer of the same gas in the same condition) of the radiation in each spectrum line. This varies not only with each form and size of tube and each current and pressure used, but with each line or part of a line studied. In this preliminary work we have striven merely for constant, reproducible conditions, and to map out the general course of phenomena. Later work for determining radiation constants must give simultaneous measurements of radiation and absorption.

Our chief results have been the determination of line intensity as a function of current and gas density in hydrogen, argon, and helium.

All lines studied increase continuously with increase of current. The primary lines of argon and hydrogen appear to be approaching a constant intensity at the highest currents, but no lines were found which showed a decrease in intensity with increase of current.

Secondary or spark lines may be produced by merely increasing the current without the use of spark or condenser. These secondary lines do not start abruptly at a large value of the internal energy even with argon, but continue along down toward zero of current. All the lines studied fall into one or the other of two distinct classes-primary lines which increase less and less rapidly with increase in current (or internal energy), and secondary lines which increase more and more rapidly with increase in current.

The secondary spectrum of helium has no prominent lines in the visible spectrum.

The potential gradients in hydrogen and helium, in a capillary $3.0 \times 30 \mathrm{~mm}$, decrease rapidly at first and then more slowly with increase of current. The curves showing line intensity are sensibly the same in form when plotted with either internal energy or current as abscissas, except that the scale is slightly enlarged near the origin. 
Line intensity as a function of gas density shows a pronounced maximum at from $\mathrm{I}$ to $4 \mathrm{~mm}$ pressure in the case of every line studied except one, $\mathrm{He} 504$.

None of the lines studied contain a constant fraction of the total internal energy of the radiating gas. In other words, no line intensity is directly proportional to the internal energy. The helium lines give the nearest approach to such a law, but even in this case, with a liberal allowance for wall losses, we are convinced that no such law holds.

The intensities of some of the lines studied remain in a constant ratio independent of the internal energy. This is true, to within the uncertainty of our measurements, of the whole of the primary spectrum of hydrogen. This means that each of these lines represents a constant fraction, not of the total internal energy, but of the whole energy of the primary spectrum. Some pairs of lines (Ar 603 and 556, He 667 and 587,504 , and 47I $\mu \mu$ ) vary alike throughout, i. e., both lines of the pair follow the same energy law with the same parameters. As a rule, intensity curves for the different lines of the same spectrum (primary or secondary) do not cross, but the red argon line (696) is a conspicuous exception.

In one spectrum (the hydrogen secondary) the logarithmic line intensities are sensibly in a constant ratio to each other (as given in the text). In no other case have we found such a relation to hold to within the uncertainty in our results.

Washington, August 6, igio. 